\title{
Qualidade de vida e condições ergonômicas em trabalhadores de um laboratório de saúde pública
}

\section{Worker's quality of life and ergonomic conditions in a public health laboratory \\ Calidad de vida y condiciones ergonómicas de trabajadores de un laboratorio de salud pública}

\author{
Hilda Aparecida Felício iD \\ Sandra Augusto Escola Técnica - São José do Rio Preto - (SP) - Brasil
}

Susilene Maria Tonelli Nardi iD

Instituto Adolfo Lutz de São José do Rio Preto - São José do Rio Preto - (SP) - Brasil

Pryscilla Mychelle da Silva Paula iD

Consultório de Cirurgia da Mão - São José do Rio Preto (SP) - Brasil

Heloisa da Silveira Paro Pedro (iD

Instituto Adolfo Lutz de São José do Rio Preto - São José do Rio Preto - (SP) - Brasil

Vânia Del'Arco Paschoal iD
Faculdade de Medicina de São José do Rio Preto - São José do Rio Preto - (SP) - Brasil

\section{RESUMO}

Objetivo: Avaliar a qualidade de vida, a presença de dor e as condições ergonômicas dos profissionais de um laboratório de saúde pública. Métodos: Estudo transversal e descritivo que avaliou 49 (90,7\%) profissionais de um laboratório de saúde pública, entre julho de 2014 e setembro de 2015, por meio de dados sociodemográficos e dos protocolos Short Form Health Survey (SF-36), Rapid Upper Limb Assessment (RULA) e Escala Visual Analógica da Dor (EVA). Para a análise descritiva das variáveis clínicas e sociodemográficas e a associação dos dados, utilizou-se o EPI Info, versão 7.2, considerando significantes p<0,05. O software Ergolândia, versão 6.0, analisou o resultado/escore Rapid Upper Limb Assessment (RULA). Resultados: Predomínio do sexo feminino $(93,1 \%)$, casadas $(57,1 \%)$, com média de idade 42,7(DP 13,4), 15 anos de estudo e renda acima de três salários $(61,2 \%)$. Os participantes que apresentaram baixa qualidade de vida relacionada à "saúde mental" tiveram maior risco ergonômico avaliado pelo RULA $(p<0,05)$. Nenhum participante apresentou postura laboral aceitável. Todos $(100 \%)$ tinham algum grau de dor. As dores moderada e intensa têm relação de forma negativa com a qualidade de vida nos seguintes domínios do SF-36: "estado geral de saúde", "dor," "vitalidade" e "saúde mental" $(p<0,05)$. Conclusão: Todos os participantes apresentam alguma dor, independente da idade ou da função. A saúde física autorrelatada e os escores obtidos por meio da EVA revelaram que as dores moderada e intensa influenciam de forma negativa a qualidade de vida e, de acordo com o RULA, nenhum participante apresenta postura laboral aceitável.

Descritores: Ergonomia; Qualidade de Vida; Postura; Dor; Pessoal de Saúde.

\section{ABSTRACT}

Objective: To assess quality of life, presence of pain and ergonomic conditions among professionals in a public health laboratory. Methods: This descriptive cross-sectional study assessed 49 (90.7\%) public health laboratory professionals between July 2014 and September 2015 using sociodemographic data and the Short Form Health Survey (SF-36), the Rapid Upper Limb Assessment (RULA), and the Visual Analog Scale (VAS) for pain assessment. The descriptive analyses of the clinical and sociodemographic variables and the analyses of associations between the data were performed on EPI Info 7.2 using a significant threshold of $p<0.05$. Ergolândia 6.0 software analyzed the Rapid Upper Limb Assessment (RULA) result/score. Results: There was a predominance of women (93.1\%), married individuals (57.1\%), individuals with a mean of 15 years of study, individuals with an income above 3 wages (61.2\%), and the mean age was 42.7 (SD 13.4). The participants who exhibited poor mental health-related quality of life were at a higher ergonomic risk assessed by the RULA $(p<0.05)$. None of the participants presented an acceptable working posture. All the participants (100\%) reported some degree of pain. Moderate and severe pain were negatively related to quality

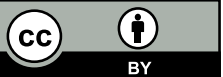


of life in the following SF-36 domains: "general health status", "pain", "vitality" and "mental health" (p<0.05). Conclusion: All the participants reported some pain regardless of age and job. Self-reported physical health and the VAS scores revealed that moderate and severe pain negatively influenced quality of life, and, according to the RULA, none of the participants presented an acceptable working posture.

Descriptors: Ergonomics; Quality of Life; Posture; Pain; Health Personnel.

\section{RESUMEN}

Objetivo: Evaluar la calidad de vida, la presencia de dolor y las condiciones ergonómicas de los profesionales de un laboratorio de salud pública. Métodos: Estudio transversal y descriptivo que evaluó 49 (90,7\%) profesionales de un laboratorio de salud pública entre julio de 2014 y septiembre de 2015 a través de datos sociodemográficos y de los instrumentos Short Form Health Survey (SF-36), la Rapid Upper Limb Assessment (RULA) y la Escala Visual Analógica del Dolor (EVA). Se utilizó el EPI Info versión 7.2 para el análisis descriptivo de las variables clínicas y sociodemográficas y la asociación de los datos considerando significantes la $p<0,05$. Con la versión 6.0 del software Ergolândia se analizó el resultado/puntuación de la Rapid Upper Limb Assessment (RULA). Resultados: Predominio del sexo femenino (93,1\%), casadas (57,1\%), con la media de edad de 42,7 años (SD 13,4), 15 años de estudio y con la renta de más de tres sueldos $(61,2 \%)$. Los participantes que presentaron baja calidad de vida relacionada a la "salud mental" tuvieron más riesgo ergonómico evaluado por la RULA ( $p<0,05)$. Ningún participante presentó la postura laboral aceptable. Todos (100\%) tenían algún grado de dolor. Los dolores moderado e intenso tiene relación de manera negativa con la calidad de vida para los dominios del SF-36 a continuación: "estado general de salud", "dolor," "vitalidad" $y$ "salud mental" $(p<0,05)$. Conclusión: Todos los participantes presentaron algún dolor independiente de la edad o la función. La salud física auto relatada y las puntuaciones obtenidas por la EVA han revelado que los dolores moderado e intenso influyen de manera negativa para la calidad de vida y según la RULA ningún participante presenta la postura laboral aceptable.

Descriptores: Ergonomía; Calidad de Vida; Postura; Dolor; Personal de Salud.

\section{INTRODUÇÃO}

A Organização Internacional do Trabalho (OIT), diante das inadequadas condições de trabalho oferecidas aos trabalhadores na saúde pública de muitos países, tem, desde a década de 40, considerado o problema como tema de discussão e feito recomendações referentes à higiene e segurança com a finalidade de ajuste das condições de trabalho desses profissionais ${ }^{(1)}$.

No Brasil, 2,4\% apresentam diagnóstico médico de doenças osteoneuromusculares relacionadas ao trabalho. Esse percentual é maior na Região Sul, ficando em 3,9\%, e os menores percentuais ficam nas regiões Nordeste $(1,4 \%)$ e Norte $(0,7 \%)$. As mulheres são mais afetadas $(3,3 \%)$ que os homens $(1,5 \%)$, e a classe economicamente ativa, dos 30 aos 59 anos, é a faixa etária que mais apresenta doença do sistema osteoneuromuscular, assim como as que possuem nível superior completo $(3,8 \%)^{(2)}$.

O controle e a estabilidade postural estão associados às funções dos sistemas visual, proprioceptivo e vestibular e ao controle neuromuscular ${ }^{(3)}$. Essas condições, quando insatisfatórias estão relacionadas a fatores biológicos, físicos, químicos, psicossociais e ergonômicos, constituindo-se em um problema de saúde pública mundial(4).

A postura correta é uma habilidade importante para uma adaptação favorável do ser humano, e permite não só movimentar-se e reconhecer-se no espaço, mas também desencadear e dar sequência aos seus gestos ${ }^{(5)}$. Nesse sentido, entende-se que a postura no ambiente de trabalho e a maneira pela qual as partes do corpo são orientadas durante a realização das atividades laborais podem desencadear alterações corporais que são refletidas pela dor musculoesquelética( ${ }^{(6,7)}$.

As queixas de desconfortos musculoesqueléticos e a influência de fatores de risco individuais e/ou psicossociais podem ter um papel significativo no desenvolvimento de lesões por esforços repetitivos (LER), também conhecida como doenças osteoneuromusculares relacionadas ao trabalho (DORT)(8), que se caracterizam por lesões de músculos, tendões, fáscias e nervos, com sintomas de dor, parestesia e sensação de peso. Baseado nesse conjunto de situações surge a ergonomia, que pode ser definida como o trabalho interprofissional, baseado num conjunto de ciências e tecnologias, que procura o ajuste mútuo entre o ser humano e seu ambiente de trabalho de forma confortável e produtiva( ${ }^{(9)}$. Existe uma relação comprovada entre as posturas estáticas ou com pouco movimento e as desordens musculoesqueléticas e a presença de dor em diversas profissões ${ }^{(10)}$.

Para um efetivo programa de prevenção de doenças ocupacionais, há de se estabelecer um comprometimento da gerência e direção no sentido de estimular a participação dos trabalhadores para a solução dos problemas, 
minimizando afastamentos médicos, acidentes e lesões. Assim, os trabalhadores devem ser corresponsáveis pela promoção da sua própria saúde ${ }^{(11)}$.

Os estudos sobre saúde ocupacional, em sua maioria, estão centrados em ambientes nos quais o profissional da área é contratado para realizar adequações ergonômicas ou laborais. O diferencial do presente estudo é o fato de ser realizado em ambiente laboratorial do setor público, em que os profissionais que transitam têm pouco conhecimento dessa necessidade ou dessa área de atuação. Diante dos fatos apresentados, este estudo intencionou avaliar a qualidade de vida, a presença de dor e as condições ergonômicas dos profissionais de um laboratório de saúde pública.

\section{MÉTODOS}

Trata-se de um estudo transversal e descritivo, com análise quantitativa do tipo inquérito, realizado com todos os profissionais de um laboratório de saúde pública de um município de grande porte no estado de São Paulo, Brasil, de referência para 102 municípios da região.

A população estudada foi composta por todos os profissionais do referido laboratório que estavam na ativa no período da coleta de dados, sendo excluídos aqueles que não aceitaram participar e os que estavam em férias ou afastamento. A coleta de dados ocorreu entre julho de 2014 e setembro de 2015, e utilizou-se um instrumento com quatro partes, dividido em três momentos: entrevista, observação da postura, formulário de qualidade de vida e postura.

Os pesquisadores, um(a) fisioterapeuta e um(a) terapeuta ocupacional, realizaram a observação e registro da postura de cada um dos profissionais durante a realização de suas funções pelo período de 20 minutos. Fotos foram tiradas para análise, em especial quanto ao posicionamento do pescoço, ombro, braço, cotovelo, antebraço, punho, tronco e pernas. A coleta de dados ocorreu no período de quatorze meses, divididos em três momentos: 1 - entrevista, observação da postura, formulário de qualidade de vida, análise da postura e da dor; 2 - educação permanente; e 3 - orientação individual e reavaliação da postura. Os autores elaboraram e utilizaram um protocolo de dados gerais, com questões demográficas, e um de condição de saúde, com questões fechadas.

Para a avaliação da postura, utilizou-se o modelo Employee Assessment Worksheet do Rapid Upper Limb Assessment (RULA), instrumento que fornece uma avaliação rápida da carga imposta ao sistema musculoesquelético e é um componente da análise ergonômica geral da tarefa executada ${ }^{(12)}$. O método RULA, proposto em $1993^{(12)}$, tem o intuito de possibilitar um estudo ergonômico de ambientes de trabalho propensos ao surgimento de danos à saúde relacionados aos membros superiores e às posturas corporais, e é acompanhado de tabelas que facilitam a avaliação da submissão do operário aos fatores de risco ${ }^{(12)}$. A avaliação de risco é feita a partir de uma observação sistemática dos ciclos de trabalho, pontuando as posturas do tronco, pescoço, pernas, carga, braços, antebraços e punhos $^{(12)}$. Além disso, o RULA permite obter um julgamento da sobrecarga biomecânica dos membros superiores, membros inferiores e do pescoço em uma tarefa ocupacional.

O RULA gera uma lista de escores para posturas específicas e classifica a postura de atividade do indivíduo por meio de uma escala de 1 a 7 , em que os escores mais altos indicam um maior risco biomecânico de desenvolver lesões. Para o resultado/escore do RULA, utilizou-se o software Ergolândia, versão 6.0, desenvolvido pela empresa FBF Sistemas, criado a partir da junção de conceitos e técnicas utilizadas em análises ergonômicas do trabalho, sendo utilizado por 26 ferramentas ergonômicas, incluindo o RULA, para avaliação e melhoria dos postos de trabalho. Destinado especialmente a ergonomistas, fisioterapeutas e àqueles da área da saúde ocupacional. Os resultados do RULA são estratificados em escores: 1-2 é considerado "Aceitável"; 3-4, necessidade de "Investigar mais"; 5-6, "Investigar mais e mudar em breve"; e 7, que indica "Investigar e mudar imediatamente"(12).

Para avaliação da dor, utilizou-se a Escala Visual Analógica da Dor (EVA), um instrumento que auxilia na aferição da intensidade da dor no indivíduo ${ }^{(13)}$ e é dividida em duas partes: de zero a 3 a dor é considerada leve; mais de 4, dor moderada e intensa.

Para o reconhecimento da qualidade de vida, que inclui as condições de vida e saúde, foi utilizado o Short Form Health Survey (SF-36), versão em português, validado do Medical Outcomes Study 36 - Item Short Form Health Survey ${ }^{(14)}$. É um questionário genérico, com conceitos não específicos para uma determinada idade, doença ou grupo de tratamento, e que permite comparações entre diferentes patologias e tratamentos. Possui questionário multidimensional formado por 36 itens, englobados em 8 escalas: capacidade funcional (10 itens), aspectos físicos (4 itens), dor ( 2 itens), estado geral de saúde (5 itens), vitalidade (4 itens), aspectos sociais (2 itens), aspectos emocionais ( 3 itens), saúde mental (5 itens) e mais uma questão de avaliação comparativa entre as condições de saúde atual com a de um ano atrás. Avalia tanto aspectos negativos de saúde (doença ou enfermidade), como aspectos positivos (bem-estar). Os dados são avaliados a partir da transformação das respostas em escala, na qual 100 é o melhor resultado e 0 é o pior de cada componente, não havendo um único valor que resuma toda a avaliação, sendo analisada cada dimensão em separado(14). 
A partir do levantamento das necessidades dos participantes, realizou-se um grupo educativo de 6 horas, dividindo os participantes em três encontros de 2 horas cada, no próprio laboratório, em datas e períodos diferentes, apresentando-os discussões acerca de postura adequada. Após um mês, entregou-se material educativo.

Os dados foram inseridos em planilha Excel e, para a análise, utilizou-se o software EPI INFO, versão 7.2. As variáveis clínicas e sociodemográficas foram apresentadas em frequência, média e desvio padrão. Os resultados do RULA foram estratificados em dois grupos. Considerou-se grupo 1 quando a pontuação variou de 1 a 4 , isto é, "aceitável" e "investigar mais", e inseridos no grupo 2 quando os escores ficaram entre 5 a 7 , ou seja, "investigar mais e mudar em breve" e "investigar e mudar imediatamente". Para verificar a associação da qualidade de vida, extraiu-se a média dos domínios do SF-36 para cada um dos escores estratificados do RULA e EVA, e utilizou-se o teste estatístico ANOVA ou Mann-Whitney. Para verificar associação entre as variáveis categóricas, utilizou-se o teste qui-quadrado ou o teste exato de Fisher, considerando significantes valores $p<0,05$.

Este estudo recebeu aprovação do Comitê de Ética em Pesquisa da Faculdade de Medicina de São José do Rio Preto (FAMERP), Protocolo n. ${ }^{\circ} 457.271$, segundo o termo da Resolução n. ${ }^{\circ}$ 466/2012 do CEP/CONEP/CNS/MS, que regulamenta os aspectos éticos da pesquisa envolvendo seres humanos. Todos os participantes assinaram 0 Termo de Consentimento Livre e Esclarecido.

\section{RESULTADOS}

Foram entrevistados $49(90,7 \%)$ profissionais, do total de 54 existentes no laboratório. Do total de entrevistados, $46(93,1 \%)$ eram do sexo feminino. A idade variou de 22 a 65 anos, média de 42,7 (DP 13,4); 28 (57,1\%) tinham companheiro e a maioria não consumia álcool $(77,5 \%)$ ou tabaco $(93,9 \%)$. Quanto à educação formal, $22(44,8 \%)$ participantes estudaram de 17 a 27 anos. A renda pessoal de 19 (38,8\%) funcionários era de até três salários mínimos e de $30(61,2 \%)$, mais de três salários.

Em relação à atuação profissional, 20 (40,8\%) exerciam o cargo de pesquisadores científicos ou assistentes técnicos de apoio à pesquisa científica e tecnológica ou auxiliar de apoio à pesquisa científica e tecnológica; 10 $(20,4 \%)$ profissionais atuavam como bolsistas; $14(28,6 \%)$ encaixavam-se nos cargos de técnicos/auxiliares de laboratório, técnicos/auxiliares de enfermagem e agente técnico de assistência à saúde; $04(8,1 \%)$ eram do setor administrativo ou diretoria e $01(2,1 \%)$ era químico.

Em resposta à pergunta sobre "ter alguma doença" e/ou "distúrbio nos dias atuais", 38 (77,5\%) responderam que não tinham problemas e $11(22,5 \%)$ responderam que sim. Da mesma forma, perguntou-se sobre a saúde física nos dias atuais, no último mês e no último ano, e se obteve as seguintes respostas: 31 (63,3\%) participantes relataram estar com saúde "moderada" no último mês e $31(63,3 \%)$ no último ano. Em relação à saúde mental e emocional, $30(61,2 \%)$ pessoas as julgaram "moderada" no último mês e ano. Ao serem questionados se receberam algum tratamento de saúde no último ano, $15(30,6 \%)$ disseram que sim. Dos entrevistados, $04(8,2 \%)$ passaram por hospitalização no último ano e $01(2,0 \%)$ usava adaptação/órtese para punho, com tala bilateral, com o duplo papel de inibir e estabilizar forças durante o movimento de preensão, "por indicação médica".

Os achados da avaliação pelo RULA estão apresentados na Tabela I. Os valores obtidos por meio do instrumento EVA revelaram que a presença da dor é constante em todos os participantes, sendo $27(55,1 \%)$ com dor leve, 21 $(42,8 \%)$ com dor moderada e $1(2,1 \%)$ com dor intensa. Os resultados do RULA e da EVA revelaram que o risco ergonômico não se associou com a intensidade da dor, $p=0,60$.

Em relação ao SF-36, que inclui as condições de vida e saúde, o escore da maioria dos participantes foi superior a 70 pontos, indicando parâmetro bom de qualidade de vida, porém os componentes que avaliam a qualidade de vida sob os aspectos da "dor" e da "vitalidade" obtiveram médias abaixo de 70 pontos. (Tabela II)

Tabela I - Resultados da postura avaliados pelo RULA - Rapid Upper Limb Assessment de profissionais de um laboratório de saúde pública. São José do Rio Preto, São Paulo, 2014-2015. (n=49)

\begin{tabular}{|c|c|c|c|c|}
\hline Nível de ação & Intervenção & Pontuação & $\mathbf{n}$ & $\%$ \\
\hline 1 & Postura aceitável & 1 ou 2 & 0 & 0 \\
\hline 2 & $\begin{array}{l}\text { Deve-se realizar uma observação } \\
\text { Podem ser necessárias mudanças }\end{array}$ & 3 ou 4 & 29 & 59,2 \\
\hline 3 & $\begin{array}{l}\text { Deve-se realizar uma investigação } \\
\text { Devem ser introduzidas mudanças }\end{array}$ & 5 ou 6 & 18 & 36,7 \\
\hline 4 & Devem ser introduzidas mudanças imediatamente & 7 ou mais & 02 & 4,1 \\
\hline
\end{tabular}


Tabela II - Distribuição da média da pontuação da qualidade de vida avaliada pelo Short Form Health Survey (SF36) segundo os componentes que o constituem. São José do Rio Preto, São Paulo, 2014-2015. (n=49)

\begin{tabular}{ll}
\hline Escalas ou componentes & $\begin{array}{c}\text { Avaliação } \\
\text { média (dp) }\end{array}$ \\
\hline Capacidade funcional & $80,9(19,3)$ \\
Aspectos físicos & $86,7(22,3)$ \\
Dor & $68,3(22,1)$ \\
Estado geral de saúde & $76,4(20,0)$ \\
Vitalidade & $66,6(18,9)$ \\
Aspectos sociais & $82,6(21,2)$ \\
Aspectos emocionais & $85,8(24,3)$ \\
Saúde mental & $73,3(20,7)$ \\
\hline
\end{tabular}

A ingestão de bebida alcoólica $(p=0,71)$ e o hábito de fumar $(p=0,31)$ não interferiram no domínio "estado geral da saúde", avaliado pelo SF-36. Já a saúde física autorrelatada como moderada, ruim ou muito ruim no último mês e no último ano teve influência negativa sobre o estado geral da saúde $(p<0,05)$. No SF-36, o domínio "vitalidade" foi considerado bom ou ótimo por $77,6 \%(n=38)$ dos entrevistados, e os que estão acima dos 43 anos parecem apresentar mais vitalidade que os mais jovens $(p=0,07)$.

No domínio "aspectos emocionais", 42 (85,7\%) profissionais consideraram esse fator bom ou ótimo, independente do cargo que ocupavam. A média dos anos de educação formal não influencia na qualidade do domínio "saúde mental" $(p=0,28)$. A dor moderada e intensa influencia negativamente a qualidade de vida relacionada aos seguintes domínios do SF-36: "estado geral de saúde" ( $p=0,03)$, "dor" $(p=0,002)$, "vitalidade" $(p=0,04)$ e "saúde mental" $(p=0,02)$. (Tabela III)

Na Tabela IV, profissionais que apresentaram baixa qualidade de vida no domínio "saúde mental" apresentaram pior condição ergonômica ao serem avaliados pelo RULA $(p<0,05)$.

Tabela III - Distribuição da média da pontuação da qualidade de vida avaliada pelo Short Form Health Survey (SF36) segundo o resultado da Escala Visual Analógica da Dor. São José do Rio Preto, São Paulo, 2014-2015. (n=49)

\begin{tabular}{lccc}
\hline \multirow{2}{*}{ Domínios da qualidade de vida - SF-36 } & Escala visual analógica da dor (EVA) & \multirow{2}{*}{ Valor-p* } \\
\cline { 2 - 3 } & Leve & Moderada e intensa & 0,22 \\
Capacidade funcional & 84,62 & 76,36 & 0,15 \\
Aspectos físicos & 90,74 & 81,81 & $0,002^{*}$ \\
Dor & 79,24 & 54,95 & $0,03^{*}$ \\
Estado geral de saúde & 81,88 & 69,77 & $0,04^{*}$ \\
Vitalidade & 71,29 & 80,90 & 0,60 \\
Aspectos sociais & 84,11 & 81,13 & 0,33 \\
Aspectos emocionais & 88,88 & 67,09 & $0,02^{*}$ \\
Saúde mental & 78,37 & & \\
\hline
\end{tabular}

${ }^{*}$ ANOVA test

Tabela IV - Distribuição da média da pontuação da qualidade de vida avaliada pelo Short Form Health Survey (SF36) segundo o resultado do RULA - Rapid Upper Limb Assessment. São José do Rio Preto, São Paulo, $2014-2015$. $(n=49)$

\begin{tabular}{lccc}
\hline \multirow{2}{*}{ Domínios da qualidade de vida SF-36 } & \multicolumn{2}{c}{ Risco ergonômico - RULA } \\
\cline { 2 - 3 } & $\begin{array}{c}\text { Aceitável, porém } \\
\text { investigar mais }\end{array}$ & $\begin{array}{c}\text { Fazer alterações e introduzir mudanças } \\
\text { em breve e/ou imediatamente }\end{array}$ & Valor-p* \\
\hline Capacidade funcional & 82,24 & 79,00 & 0,56 \\
Aspectos físicos & 88,79 & 83,75 & 0,44 \\
Dor & 71,02 & 64,45 & 0,31 \\
Estado geral de saúde & 78,13 & 74,00 & 0,48 \\
Vitalidade & 68,62 & 63,75 & 0,38 \\
Aspectos sociais & 86,63 & 76,92 & 0,11 \\
Aspectos emocionais & 87,34 & 83,70 & 0,61 \\
Saúde mental & 78,34 & 66,00 & $0,03^{*}$ \\
\hline
\end{tabular}

*ANOVA test 
Diante dos dados encontrados e como parte do estudo, realizou-se adicionalmente um grupo de autocuidado, ocorrendo encontros por meio de aulas expositivas para pequenos grupos de participantes, direcionadas para esclarecer sobre o cuidado e a importância do autoconhecimento corporal, os períodos de descanso e alongamentos. Ainda, distribuíram-se fôlderes com instruções sobre a prevenção de lesões osteoneuromusculares e a postura correta que deveria ser adotada, um material didático elaborado especialmente para os problemas encontrados. Por fim, considerando-se a necessidade de intervenções futuras, realizou-se ajustes no mobiliário, como, o uso de material para elevar monitores de computadores, apoios para os pés e trocas de cadeiras.

\section{DISCUSSÃO}

O presente estudo analisou os dados demográficos, a qualidade de vida (QV), a postura e a dor de trabalhadores de um laboratório de saúde pública, contribuindo, de certa forma, para a prevenção de lesões osteoneuromusculares por meio da conscientização para a importância de adotarem uma postura correta e de aperfeiçoarem sua flexibilidade, sua coordenação e sua resistência à fadiga no final da jornada de trabalho ${ }^{(15)}$.

O sexo feminino prevaleceu no ambiente laboratorial estudado. As mulheres apresentam maior predisposição às LER/DORT, provavelmente pela dupla jornada de trabalho, do tônus mais frágil e menor densidade e tamanho dos ossos $^{(16,17)}$. Ademais, a mulher tem ocupado cada vez mais novos espaços de atuação no mercado de trabalho(18).

A população estudada caracterizou-se por ser adulta jovem, de indivíduos que estão a caminho do envelhecimento e, para que as estruturas osteoneuromusculares envelheçam com saúde, é imprescindível adotar diariamente posturas adequadas e realizar exercícios com orientação de um profissional da área ${ }^{(16,19)}$. Envelhecer de forma saudável é mostrarse ativo com suas capacidades funcionais, que tendem a diminuir com o passar dos anos, porém é a capacidade intrínseca, física e mental, associada ao ambiente em que as pessoas vivem, bem como suas interações nele, que traduzem uma vida saudável(20).

O método RULA contém uma relação comprovada entre as posturas estáticas, sentadas por longo período ou com pouco movimento, e a ocorrência de desordens musculoesqueléticas em diversas profissões ${ }^{(10)}$. A avaliação individual dos profissionais pelo RULA mostrou que a maioria dos avaliados necessita de investigação sobre sua postura e que nenhum profissional possuía uma postura "aceitável". A falta de manutenção do alinhamento corporal pode gerar patologias ocupacionais, resultantes de maior sobrecarga muscular nas regiões afetadas ${ }^{(7)}$.

Esses achados ressaltam a importância de uma postura adequada, aquela em que as articulações estão em posição neutra: o centro de gravidade das partes do corpo envolvidas na execução da tarefa está alinhado verticalmente, passando o mais próximo possível dos eixos de rotação gerados pelas articulações ${ }^{(7)}$. Assim, a manutenção de uma postura inadequada propicia determinadas adaptações estruturais do tecido muscular estriado esquelético ${ }^{(21)}$.

Todavia cabe destacar que problemas relacionados ao sistema osteomuscular no trabalho, geralmente, ocorrem quando há desequilíbrio entre a demanda física do trabalho e a capacidade física do profissional, uma vez que a anatomia do sistema musculoesquelético é desenvolvida para se movimentar, de forma que possibilita ao profissional realizar as atividades, porém é necessário repouso para recuperação e para não comprometer sua função(22).

Em relação à pontuação do instrumento EVA, os profissionais que participaram deste estudo revelaram que, em algum momento do dia, apresentam dor. Assim se faz necessária mudança na postura e no mobiliário, a introdução da ginástica laboral e a gestão no tempo de permanência na mesma posição. Estudo conclui que, quando o trabalhador refere ter dor musculoesquelética em um ponto específico do corpo, há associação da dor em outros locais do corpo, ou seja, dor multisites ${ }^{(17)}$.

As dimensões do posto de trabalho podem forçar o profissional a adotar posturas, a suportar certas cargas e a se comportar de modo a causar afecções musculoesqueléticas, contribuindo para a geração de dor, que limita 0 profissional na execução de movimentos e, consequentemente, leva à perda de força muscular ${ }^{(23)}$.

Encontrou-se uma boa condição da QV nos profissionais avaliados no presente estudo nos domínios do instrumento SF-36, e há de se ressaltar que os componentes que avaliam a qualidade de vida sob os aspectos de "dor" e "vitalidade" obtiveram médias abaixo do considerado aceitável. Nesse contexto, recomenda-se que, uma vez realizado o diagnóstico dos possíveis riscos que o ambiente de trabalho pode exercer sobre a QV, seja realizada uma intervenção e/ou replanejamento no ambiente físico, na organização e nos postos de trabalho. Como proposta, sugerem-se ajustes no desenho dos equipamentos, como cadeira ergonômica, mesa adequada para o tipo de trabalho ou para as dimensões corporais do trabalhador, e que esses equipamentos sejam adequados a diferentes requisitos da tarefa, com vistas a uma função ergonômica mais adequada.

Inclui-se aqui a proposta da ginástica laboral e o incentivo à prática de exercícios físicos rotineiros ${ }^{(24)}$, o que reflete na melhoria da qualidade de vida no trabalho e pode contribuir consideravelmente com o aumento da 
produtividade ${ }^{(25)}$. Tais ajustes podem minimizar os efeitos deletérios da postura sentada ou em pé para as estruturas musculoesqueléticas. As condições de trabalho podem ser um fator de descontentamento e piora do estado de saúde, mas também podem gerar satisfação e bem-estar ${ }^{(24,26)}$.

Em relação à qualidade de vida, variáveis diversas podem ser incluídas, como os fatores emocionais, ligados ou não ao cargo que os trabalhadores exercem ${ }^{(27)}$. Como exemplo, cita-se a associação entre sonolência diurna excessiva e baixa vitalidade. Escores baixos no domínio "dor" -do instrumento SF-36 podem estar associados a menor capacidade de deambulação e a dificuldades em realizar as atividades diárias e suas tarefas no trabalho ${ }^{(28)}$. A dor impacta na diminuição da autonomia, que resulta em problemas sociais e econômicos, reduzindo, inclusive, a sua capacidade de ganho financeiro e físico ${ }^{(28)}$.

O autorrelato da saúde física como moderada, ruim ou muito ruim no último mês e no último ano teve influência negativa sobre o estado geral da saúde dos participantes investigados na atual pesquisa. A qualidade de vida pessoal pode ser conceituada como uma escolha intencional de estilo de vida, caracterizada por responsabilidade pessoal, equilíbrio e máximo aprimoramento do bem-estar físico, mental, espiritual e social ${ }^{(29)}$.

As orientações individuais sobre autocuidado relacionados à dor e problemas na postura na atividade laboral no momento da entrega do fôlder reforçam as intervenções, minimizando os efeitos danosos e causando impacto positivo nas atividades dos profissionais. Porém a mudança nos hábitos de vida depende quase que exclusivamente do indivíduo que está sendo orientado, de modo que o profissional habilitado para estabelecer um programa preventivo de LER/DORT é um facilitador. As pessoas tendem a ser mais felizes na medida em que adotam um estado mental positivo e conseguem o que desejam, no entanto a qualidade de vida pode ser afetada e precisa ser considerada pelos aspectos que permeiam a saúde física, psicológica, crenças pessoais, relações sociais, nível de independência e relação com o meio ambiente ${ }^{(30)}$.

Apreende-se que este estudo teve como limitações a participação de um número reduzido de profissionais dentro do laboratório de escolha. Os pesquisadores precisaram adequar os turnos de trabalho dos entrevistados com as avaliações e orientações. Os pesquisadores encontraram limitações, de ordem gerencial, para efetivar mudanças, em especial no mobiliário. Com base nos achados, os autores realizaram ações, como a prática educativa por meio de palestras, a entrega de fôlder educativo especialmente elaborado para o público, as orientações de autocuidado sobre a adoção de mudanças diárias de hábitos, as posturas adequadas para prevenção de doenças osteoneuromusculares e o ajuste no mobiliário. Os resultados apresentados incitam novas pesquisas sobre o tema, que poderão fundamentar futuros protocolos de intervenção no ambiente laboratorial. Este estudo pode contribuir para um melhor entendimento do ambiente, das condições de trabalho e da qualidade de vida dos profissionais que atuam nos laboratórios de saúde pública e, com base nos achados, contribuir para a promoção da saúde, a prevenção de lesões osteoneuromusculares e afastamentos por doença, e a melhoria das condições de trabalho.

\section{CONCLUSÃO}

Este estudo revelou que, em algum momento do dia, todos os participantes apresentam alguma dor, independente da idade ou da função. A saúde física autorrelatada e os escores obtidos por meio da Escala Visual Analógica da Dor (EVA) revelaram que a dor moderada e intensa influenciam de forma negativa a qualidade de vida dos participantes, de acordo com o SF-36, principalmente nos domínios "estado geral de saúde", "dor", "vitalidade" e "saúde mental".

O ambiente laboratorial estudado revelou que a maioria dos participantes é do sexo feminino, com média de idade de 42,7 anos, e exercem atividades ligadas à pesquisa ou atividades práticas de laboratório. De acordo com o Rapid Upper Limb Assessment (RULA), nenhum participante apresenta postura laboral aceitável. Além disso, para 95,9\% deles há necessidade de observação e investigação, pois pode ser necessário introduzir mudanças no contexto laboral. Com a aplicação do instrumento SF-36, 70\% dos participantes apresentam qualidade de vida "boa", exceto nos domínios "dor" e "vitalidade". Os profissionais que apresentaram baixa qualidade de vida no domínio "saúde mental" também apresentaram pior condição ergonômica quando avaliados pelo RULA.

\section{CONFLITOS DE INTERESSE}

Os autores declaram não haver conflitos de interesses.

\section{CONTRIBUIÇÕES}

Hilda Aparecida Felício, Susilene Maria Tonelli Nardi e Vânia Del'Arco Paschoal contribuíram com a concepção e delineamento do estudo; a análise e interpretação dos resultados; e a redação e revisão crítica do conteúdo do 
manuscrito. Pryscilla Mychelle da Silva Paula e Heloisa da Silveira Paro Pedro contribuíram com a concepção e delineamento do estudo; e a redação e revisão crítica do conteúdo do manuscrito. Todos os autores aprovaram a versão final do manuscrito e são responsáveis por todos seus aspectos, incluindo a garantia de sua precisão e integridade.

\section{AGRADECIMENTOS}

Fundação de Apoio à Pesquisa e Extensão de São José do Rio Preto - FAPERP.

Artigo baseado na dissertação de mestrado intitulada: "Qualidade de vida, condições ergonômicas e presença de dor entre trabalhadores em um laboratório de saúde pública". Apresentada na Famerp - Faculdade de Medicina de São José do Rio Preto no ano de 2019, 48 páginas.

\section{FONTES DE FINANCIAMENTO}

Estudo financiado com recursos da Fundação de Apoio à Pesquisa e Extensão de São José do Rio Preto - FAPERP: Processo $n^{\circ}$ 044/2014. Felício HA recebeu bolsa de mestrado pelo programa de Demanda Social outorgado pela Coordenação de Aperfeiçoamento de Pessoal de Nível Superior - CAPES aprovada pelo Edital $n^{\circ}$ 002/2017 da Pós-Graduação em Enfermagem da Faculdade de Medicina de São José do Rio Preto. CAPES Processo Bolsas 18/19 23038006436/2018-19.

\section{REFERÊNCIAS}

1. Organização Internacional do Trabalho, Departamento de Políticas de Emprego. Guia para a formulação de políticas nacionais de emprego [Internet]. 2013 [acesso em 2020 Jul 15]. Disponível em: https://www.ilo.org/ wcmsp5/groups/public/---ed_emp/---emp_policy/documents/publication/wcms_214960.pdf

2. Ministério do Planejamento, Orçamento e Gestão (BR); Instituto Brasileiro de Geografia e Estatística. Pesquisa Nacional de Saúde 2013: percepção do estado de saúde, estilo de vida e doenças crônicas [Internet]. Rio de Janeiro: IBGE, 2014 (acesso em 2020 Jul 15). Disponível em: https://biblioteca.ibge.gov.br/ visualizacao/livros/liv91110.pdf

3. Lara S, Graup S, Balk RS, Teixeira LP, Farias AD, Alves GB, et al. Associação entre o equilíbrio postural e indicadores antropométricos em escolares. Rev Paul Pediatr [Internet]. 2018 [acessado em 2019 Dez 10];36(1):59-65. Disponível em: https://www.scielo.br/scielo.php?script=sci_arttext\&pid=S010305822018000100059\&lng=en

4. Zavarizzi CP, Alencar MCB. Afastamento do trabalho e os percursos terapêuticos de trabalhadores acometidos por LER/Dort. Saúde Debate [Internet]. 2018 [acessado em 2019 Nov 12]; 42(116):113-24. Disponível em: http://www.scielo.br/scielo.php?script=sci_arttext\&pid=S0103-11042018000100113\&lng=en

5. Souchard PE. Reeducação Postural global (Método do Campo Fechado). $4^{\mathrm{a}}$ ed. São Paulo: Cone; 2011.

6. Velasco-Key KM. Análisis de las posturas por instrumentadores quirúrgicos durante cirúrgia. Rev Salud Pública [Internet]. 2017 [acesso em 2019 Out 19];19(1). Disponível em: https://scielosp.org/article/ rsap/2017. v19n1/112-117/es/

7. Onety GCS, Leonel DV, Saquy PC, Silva GP, Ferreira B, Varise TG, et al. Analysis of endodontist posture utilizing cinemetry, surface electromyography and ergonomic checklists. Braz Dental J [Internet]. 2014 [acesso em 2020 Mar 26];25(6):508-18. Disponível em: https://www.scielo.br/scielo.php?script=sci_arttext\&pi $\mathrm{d}=$ S0103-64402014000600508

8. Carregaro RL, Toledo AM, Christofoletti G, Oliveira AB, Cardoso JR, Padula RS. Association between work engagement and perceived exertion among healthcare workers. Fisioter Mov [Internet]. 2013 [acesso em 2019 Out 17];26(3):579-85. Disponível em: https://www.scielo.br/scielo.php?script=sci_arttext\&pid =S0103-51502013000300011

9. Couto HA. Como implantar ergonomia na empresa, a prática dos comitês de ergonomia. Belo Horizonte: Ergo; 2002.

10. Saliba T, Machado A, Garbin A. Análise ergonômica do atendimento clínico odontológico. Revista ABENO 
[Internet]. 2016 [ acesso em 2020 Jan 27];16(3):96-105. Disponível em: https://revabeno.emnuvens.com.br/ revabeno/article/view/284

11. Teixeira VMF. Citotécnico: análise do processo de trabalho em laboratórios de citopatologia e anatomopatologia no Estado do Rio de Janeiro [tese]. Rio de Janeiro: Universidade do Estado do Rio de Janeiro; 2015.

12. McAtamney L, Nigel Corlett E. RULA: a survey method for the investigation of work related upper limb disorders. Appl Ergon [Internet]. 1993 [acesso em 2019 Jul 17];24(2):91-9. Disponível em: https://www. sciencedirect.com/science/article/abs/pii/000368709390080S?via\%3Dihub

13. Nohara SSB, Bonifácio SR, Lemos LC. Physical therapy intervention in physical stress and pain in Caregivers of children with cerebral palsy. Rev Bras Promoç Saúde [Internet]. 2017 [acesso em 2020 Jan 20];30(4):1-

7. Disponivel em: https://search.proquest.com/openview/4031bd87f91b3a30d3c181098ff6dc1b/1?pqorigsite $=$ gscholar $\& \mathrm{cbl}=2046042$

14. Ciconelli RM, Ferraz MB, Santos W, Meinão I, Quaresma MR. Tradução para a língua portuguesa e validação do questionário genérico de avaliação de qualidade de vida SF-36 (Brasil SF-36). Rev Bras Reumatol [Internet]. 1999 [acesso em 2019 Dez 11];39(3):143-50. Disponível em: https://www.ufj.br/renato_nunes/ files/2014/03/Valida\%c3\%a7\%c3\%a3o-do-Question\%c3\%a1rio-de-qualidade-de-Vida-SF-36.pdf

15. Martínez VM, Callejo- Dominguez JM, Beltrán-lasco I, Peréz-Carmona N, Abellán-Miralles G, GonzálesCabalerro G, et al. Folletos de información educativa em migraña: satisfacción percebida em um grupo de pacientes. Neurología [Internet]. 2015 [acesso em 2019 Dez 04];30(8):472-8. Disponível em: https://reader. elsevier.com/reader/sd/pii/S0213485314001182?token=34F7737EB648BBE498385FFB93C9CA99AC394FE FF34715BB8C3F9622A3F5A73EEECFB6278DCE46A4E633BF1EEC39AE15

16. Santos M, Guerreiro M, Hamada A, Santos K, Luciano L. Percepção sobre ergonomia pelos acadêmicos de Odontologia de uma faculdade privada de Imperatriz-MA. Rev Odont Araç [Internet]. 2017 [acesso em 2019 Dez 05];38(1):19-26. Disponível em: https://apcdaracatuba.com.br/revista/2017/04/TRABALHO3.PDF

17. Fernandes RCP, Pataro SMS, Carvalho RB, Burdorf A. The concurrence of musculoskeletal pain and associated work-related factors: a cross sectional study. BMC Public Health [Internet]. 2016 [acesso em 2019 Dez 05];16:628. Disponível em: https://www.ncbi.nlm.nih.gov/pmc/articles/PMC4957833/

18. Reis AH, Brito MS, Simioni P. Gerenciamento da renda familiar por jovens casais. Pensando Fam [Internet]. 2016 [acesso em 2019 Dez 04];21(2):28-44. Disponível em: http://pepsic.bvsalud.org/scielo.php?script=sci_ arttext\&pid=S1679-494X2017000200004

19. Baumert P, Lake MJ, Stewart CE, Drust B, Erskine RM. Genetic variation and exerciseinduced muscle damage: implications for athletic performance, injury and ageing. Eur J Appl Physiol. 2016;(116):1595-625.

20. Macedo MLM, Amaral AKFJ. Oficina de expressividade vocal para pessoas idosas. Rev Pesqui Cuid Fundam. 2018;10(3):208-11.

21 Makofsky HW. Coluna vertebral Terapia Manual. Rio de Janeiro: Guanabara Koogan; 2006.

22. Pacheco ES, Sousa ARR, Sousa PTM, Rocha AF. Prevalence of musculoskeletal symptoms related to nursing work in the hospital field. Rev Enferm UFPI [Internet]. 2016 [acesso em 2019 Dez 04];5(4):31-7. Disponível em: https://revistas.ufpi.br/index.php/reufpi/article/view/5387

23. Longen WC, Barcelos LP, Karkle KK, Schutz FS. Avaliação da incapacidade e qualidade de vida de trabalhadores da produção de indústrias cerâmicas. Rev Bras Med Trab [Internet]. 2018 [acesso em 2019 Dez 04];16(1):10-8. Disponível em: https://www.rbmt.org.br/details/288/pt-BR/avaliacao-da-incapacidade-equalidade-de-vida-de-trabalhadores-da-producao-de-industrias-ceramicas

24. Gomes M, Carvalho N, Nisihara R. Análise da qualidade de vida dos costureiros e sua relação com o vínculo empregatício. Rev Bras Med Trab [Internet]. 2016 [acesso em 2019 Dez 04];14(3):237-44. Disponível em: https://www.rbmt.org.br/details/117/pt-BR/analise-da-qualidade-de-vida-dos-costureiros-e-sua-relacao-com-ovinculo-empregaticio

25. Corrêa TRMFC, Cintra MMMC, Paulino TP. Avaliação da qualidade de vida de praticantes de ginástica laboral. Medicina (Ribeirão Preto, Online) [Internet]. 2017 [acesso em 2019 Dez 04];50(1):11-7. Disponível 
em: http://revista.fmrp.usp.br/2017/vol50n1/AO2-Avaliacao-da-qualidade-de-vida-de-praticantes-de-ginasticalaboral.pdf

26. Vasconcelos T, Cardoso A. Análise ergonômica e postural dos citologistas de um hospital na cidade de Fortaleza (CE). Saúde e Pesqui. 2016;9(2):333-41.

27. Ribeiro WRB, Gondim SMG, Pereira CR. Personalidade e bem-estar subjetivo de trabalhadores: moderação da regulação emocional. Arq Bras Psicol [Internet]. 2018 [acesso em 2019 Dez 04];70(1):69-85. Disponível em: http://pepsic.bvsalud.org/scielo.php?script=sci_abstract\&pid=S1809-52672018000100017\&lng=pt\&nrm=i so

28. Back Netto M, Barranco ABS, Oliveira KWK, Petronilho F. Influência dos sintomas de ansiedade e depressão na qualidade de vida de pacientes submetidos à cirurgia de coluna lombar. Rev Bras Ortop [Internet]. 2018 [acesso em 2019 Dez 04];53(1):38-44. Disponível em: https://www.scielo.br/scielo.php?pid=S0102$36162018000100038 \&$ script $=$ sci_arttext\&tlng=pt

29. Klein S, Fofonk A, Hirdes A, Jacob M. Qualidade de vida e níveis de atividade física de moradores de residências terapêuticas do sul do Brasil. Ciênc Saúde Colet [Internet]. 2018 [acesso em 2019 Dez 04];23(5):1521-30. Disponível em: https://www.scielo.br/scielo.php?script=sci_arttext\&pid $=\mathrm{S} 1413-81232018000501521$

30. Gouveia MTO, Santana HS, Santos AMR. Quality of life and welfare of nursing university students: integrative review. Rev Enferm UFPI [Internet]. 2017 [acesso em 2019 Dez 04];6(3):72-8. Disponível em: https://revistas. ufpi.br/index.php/reufpi/article/view/6074

\author{
Endereço do primeiro autor: \\ Hilda Aparecida Felício \\ SAETEC - Sandra Augusto Escola Técnica \\ Rua Boa Vista, 512 \\ Bairro: Boa Vista \\ CEP: 15025-010 - São José do Rio Preto - SP - Brasil \\ E-mail: hilda.felicio@hotmail.com \\ Endereço para correspondência: \\ Susilene Maria Tonelli Nardi \\ Instituto Adolfo Lutz de São José do Rio Preto \\ Rua: Alberto Sufredini, 2325 \\ Bairro: Jardim Maceno \\ CEP: 15060-020 - São José do Rio Preto - SP - Brasil \\ E-mail: susilene.nardi@ial.sp.gov.br
}

Como citar: Felício HA, Nardi SMT, Paula PMS, Pedro HSP, Paschoal VD. Qualidade de vida e condições ergonômicas em trabalhadores de um laboratório de saúde pública. Rev Bras Promoç Saúde. 2020;33:11017. 